\title{
Research Paper \\ The Effect of Quisinostat as the HDAC Inhibitor on Migration
}

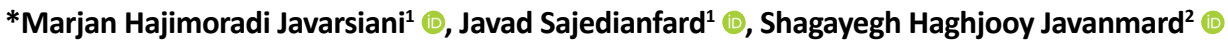 \\ 1. Department of Basic Sciences, School of Veterinary Medicine, Shiraz University, Shiraz, Iran. \\ 2. Applied Physiology Research Center, Cardiovascular Research Institute, Isfahan University of Medical Sciences, Isfahan, Iran.
}

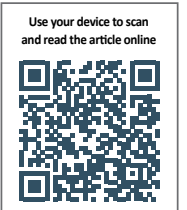

Cftation: Hajimoradi Javarsiani M, Sajedianfard J, Haghjooy Javanmard Sh. [The Effect of Quisinostat as the HDAC Inhibitor on Migration (Persian)]. Journal of Arak University of Medical Sciences (JAMS). 2021; 24(3):450-457. https://doi.org/10.32598/ JAMS.24.3.6425.1

https://doi.org/10.32598/JAMS.24.3.6425.1

Key words:

HDAC, Quisinostat, Cancer cells, Migration

\section{ABSTRACT}

Article Info:

Received: 17 Jan 2021 Accepted: 24 Apr 2021 Available Online: 01 Aug 2021

\begin{abstract}
Background and Aim Cancer cannot be explained only by genetic alterations but involves epigenetic processes. Modifying histones by acetylation plays a key role in epigenetic regulation of gene expression and is controlled by the balance between Histone Deacetylases (HDAC) and Histone Acetyltransferases (HAT). The HDACs expression and activity could be involved in several tumorigenesis mechanisms, so their inhibition induces cancer cell cycle arrest and migration.

Methods \& Materials Quisinostat is a novel promising second-generation HDAC inhibitor class of hydroxamic acid with high cellular potency towards classes I and II HDACs. Therefore, its low IC50 ( $<0.5 \mathrm{nM})$ and bioavailability have been chosen to carry out our studies. Cancer cells were treated with Quiznos at nM200, and cell migration was measured by fluorescent microscopy.

Ethical Considerations This study was the result of a preliminary study of Shiraz University (Code: 96GCU3M1293).

Results The data showed that treatment of cancer cells with Quiznos significantly $(\mathrm{P}<0.05)$ reduced cell migration. DMSO did not affect reducing cell migration.

Conclusion In this project try to explore the possible therapeutic application of this HDAC inhibitor against colon cancer. This study showed Quisinostat exerts broad-spectrum antiproliferative activity and migration.
\end{abstract}

\section{Extended Abstract}

\section{Introduction}

\section{Background and Purpose}

olorectal cancer is the third deadliest cancer in the world [1]. Cancer cells under different environmental conditions have different survival and proliferative power, resulting from successive mutations in the cancer cell [2]. Tumor formation is related to epigenetic changes in addition to genetic differences. One of the epigenetic pathways is histone acetylation. Acetylation opens the chromatin and allows the operator to access the transcription mechanism $[3,4]$.

Histone deacetylases are a group of enzymes responsible for removing acetyl groups from the amino acid lysine. Alterations of the histone deacetylase enzyme have been observed in many cancers, so drugs that inhibit this enzyme may play a role in inhibiting cancer [5]. Quisinostat as a new drug has high potential in the treatment of tumors [6]. It has anti-neoplastic properties in nanomolar doses by inhibiting the distillation of histones [7]. It has been suggested that epigenetic changes may be a sign of cancer diagnosis. These changes after transmission may play an essential role

\section{* Corresponding Author:}

Marjan Hajimoradi Javarsiani, PhD.

Address: Department of Basic Sciences, School of Veterinary Medicine, Shiraz University, Shiraz, Iran.

Tel: +98 (313) 4256229

E-mail:marhaji@yahoo.com 
in cancer progression through gene transcription modulation, chromatin regeneration, and nuclear architecture. Alterations in acetylation or expression of HDAC have been associated with several solid tumor cancers [8].

\section{Materials and Methods}

Quizinostat was dissolved in DMSO, which is a natural analgesic and anti-inflammatory. To dissolve the drug, a safe amount was calculated. Three groups were considered for the experiment, respectively. The first group was un- treated cells, the second group was cells treated with quizinostat with a concentration of $200 \mathrm{nM}$, and the third group was cells treated with DMSO.

The cell suspension was cultured in the upper wells of the Boyden chamber assay, where 1000 cells with $50 \mu \mathrm{L}$ of culture medium were placed in each well.

Cells migrate along the membrane. Since quisinostat is dissolved in DMSO, and due to the toxicity of DMSO for cells, it was calculated that the concentration of DMSO in the well
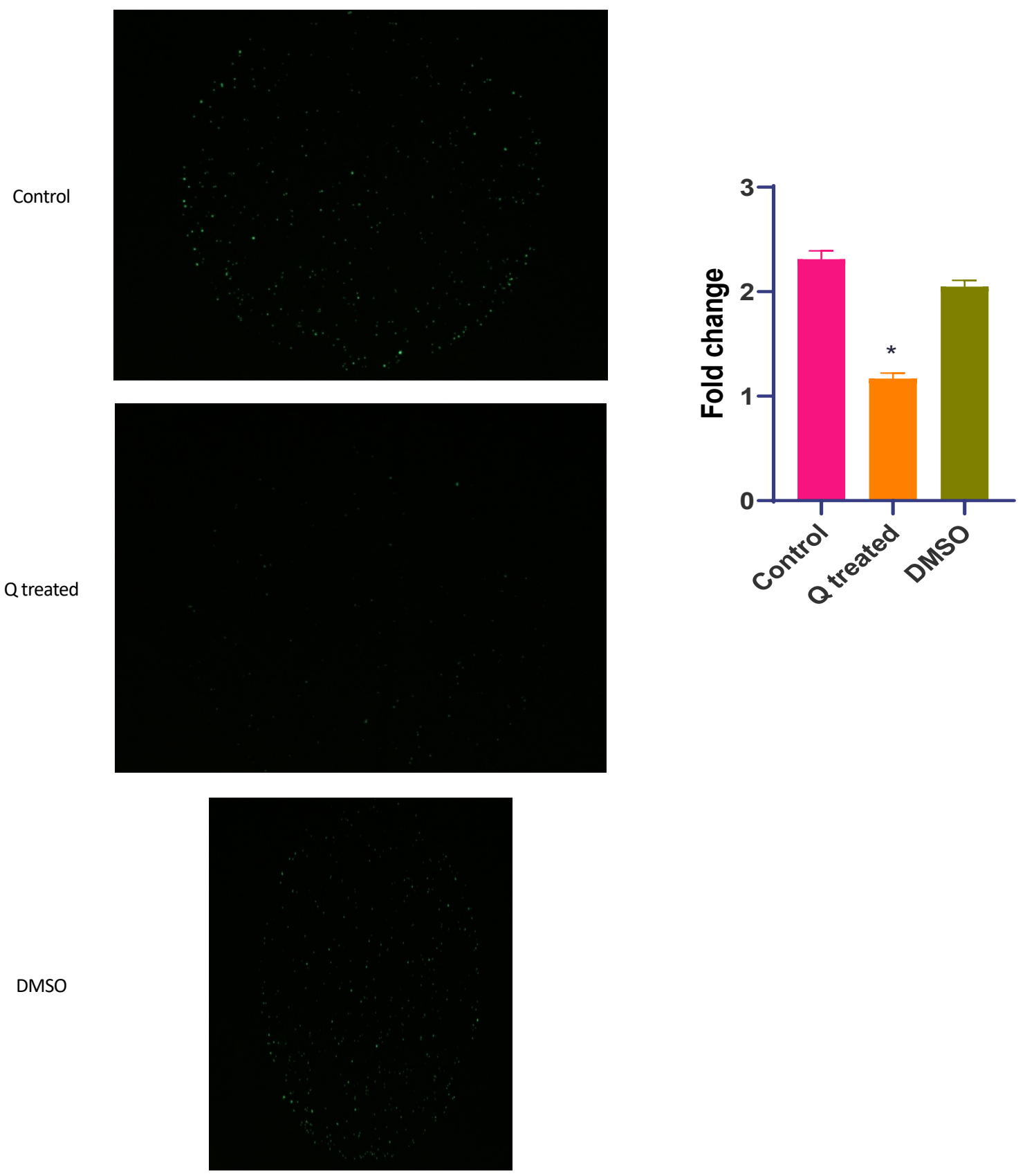

Figure 1. Cancer cells were cultured in a special plate for cell migration test 
should not exceed $0.25 \%$. To investigate the migration of treated cells to the lower wells, $225 \mu \mathrm{L}$ of culture medium was poured into each well. This experiment was measured 24 hours after treatment and repeated 3 times. To count the migration rate 24 hours after treatment, the lower part was stained with a fluorescent dye, and the number of cells in the lower well was measured with a special microscope.

\section{Results}

Before adding the cell suspension to the upper part of the cell membrane, they were kept in a culture medium without FBS for 24 hours until they reached starvation. 24 hours later, the number of cells migrating to the lower abdomen was counted. It should be noted that according to the cell size, the size of $12 \mu \mathrm{M}$ well filter holes was selected [10]. The results showed that quisinostat is a beneficial and effective drug to prevent the migration of cancer cells and prevent metastasis and spread of cancer in the long run. The figure shows that treatment of cells with quisinostat nM200 reduced cell migration by $50 \%$ (Figure 1 ).

Treatment was assessed 24 hours after cell culture and 24 hours after treatment by cell fluorescence microscopy. All experiments were repeated at least 3 times. Data were analyzed using a one-way ANOVA test. In this test, a value of 0.05 or less was considered significant.

\section{Discussion and Conclusion}

In this paper, treatment with HDACi on colorectal cell lines was investigated. The results showed that DMSO alone did not significantly affect cell migration, but treatment with quisinostat reduced the migration rate by up to $50 \%$. It can be concluded that quisinostat by histone distillation has caused chromatin compression and subsequent suppression of genes effective in the expression of proteins necessary for cell migration [8]. Previous research has also evaluated the effect of HDACi on proliferative cell pathways in cancer and found, for example, that HDACi can activate P53 [5] or increase the susceptibility of cancer cells to apoptosis [4]. Oral administration of quisinostat causes persistent $\mathrm{H} 3$ acetylation and inhibits tumor progression in colorectal tumors [3].

The histone deacetylase enzyme affects chromatin by deacetylating lysine residues; it restricts histones available at transcription sites. The adverse activity of this enzyme has been observed in a variety of cancers. This activity transforms cancer cells into CSCs and increases metastasis and cell migration because it prevents the expression of genes that lead to defective cells leading to apoptosis [9, 11]. Members of the distilling enzyme family have played a vital role in the devel- opment and progression of cancer. In recent years, by examining the expression of the gene that produces these enzymes in various types of cancer and the effect of inhibitory drugs, it has been possible to create therapeutic goals, especially new medicines in the treatment of cancers [4].

Investigation and repair of histone acetylation can play an essential role in the homeostasis and function of chromatin proteins; these molecules inhibit its function by binding zinc ions in the active site of the deacetylating enzyme [8]. Also, during the use of HDACi, this drug has an influential role in killing cancer cells due to the greater sensitivity of cancer cells to apoptosis than normal cells $[8,12]$. This study showed that quisinostat, as a histone acetylation inhibitor, could inhibit cell migration and prevent cancer metastasis.

\section{Ethical Considerations}

\section{Compliance with ethical guidelines}

This study was the result of a preliminary study of Shiraz University (Code: 96GCU3M1293).

\section{Funding}

This research did not receive any grant from funding agencies in the public, commercial, or non-profit sectors.

\section{Authors' contributions}

All authors met the standard writing standards based on the recommendations of the International Committee of Medical Journal Publishers.

\section{Conflicts of interest}

The authors declared no conflict of interest.

\section{Acknowledgements}

The authors would like to thank the Rome Health Research Center of Italy and the esteemed colleagues in the Biobank Center for their help in implementing this project. 


\title{
بروسى اثر كويزينوسثات به منزله يك مهاركنثده داستياسيون بر مهاجرت سلولى
}

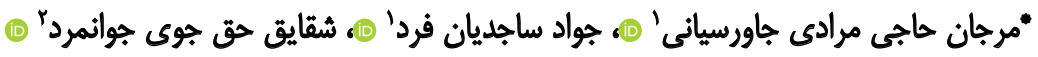

1. ا.كروه علوم يايه، دانشكده داميزشكى، دانشكاه شيراز، شيراز، ايران.

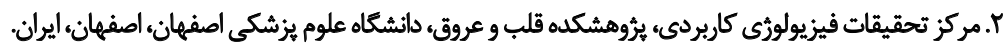

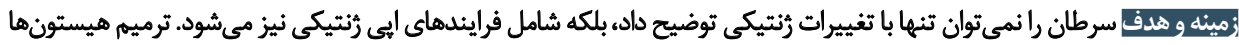

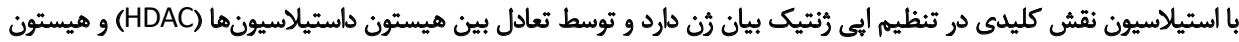

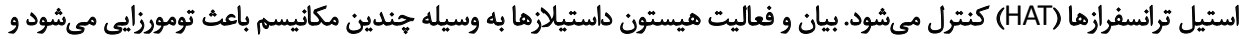

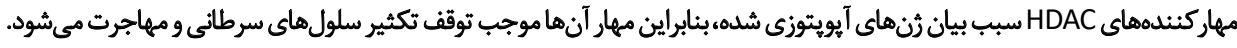

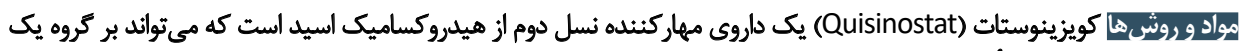

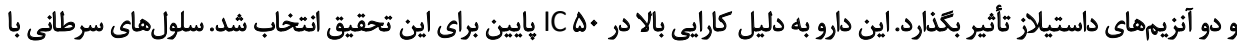

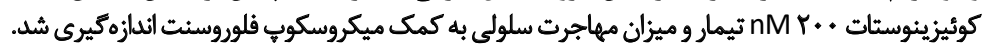

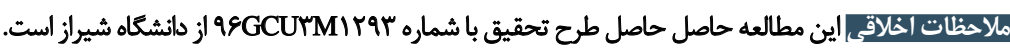

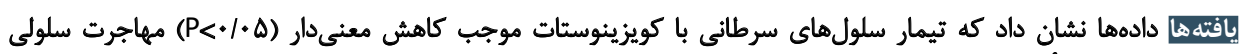

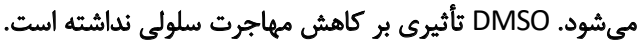

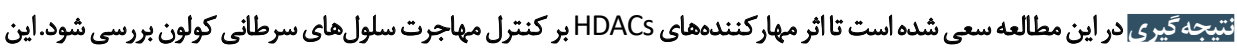

آنزيم هيستون داستيلاز در بسيارى از سرطانها از جمله سرطان

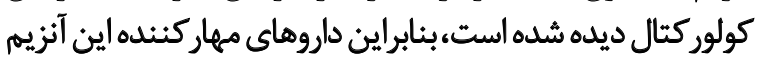

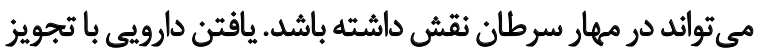

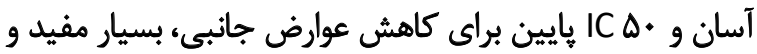

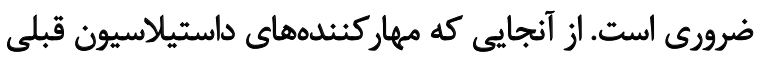

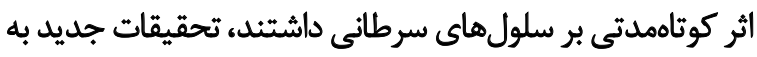

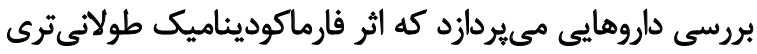

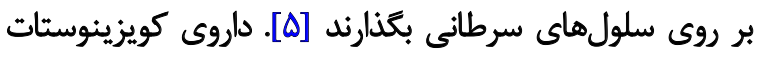

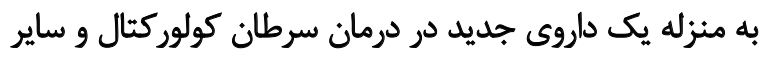

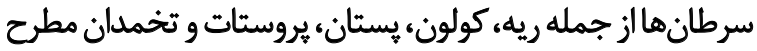

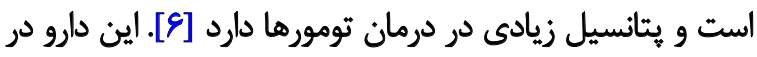

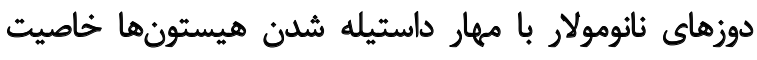

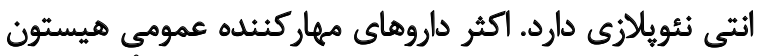

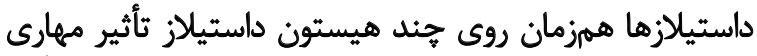

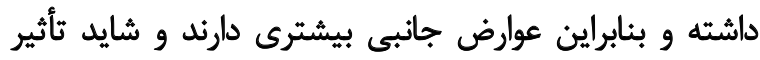

سرطان كلوركتال سومين سرطان كشنده در جهان است. اين

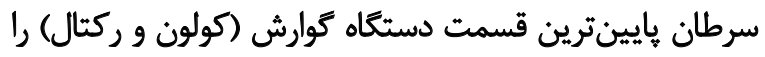

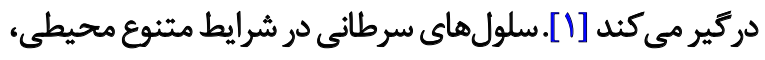

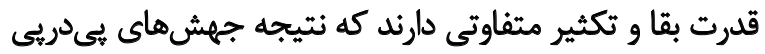

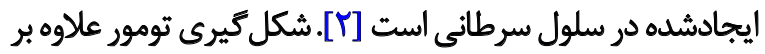

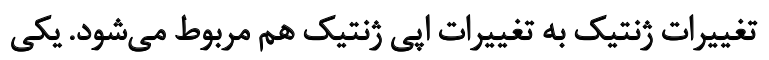

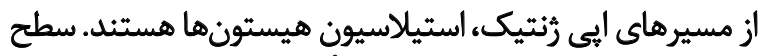

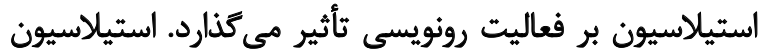

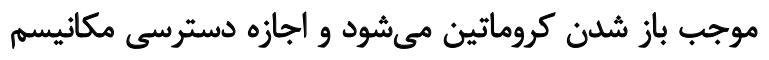

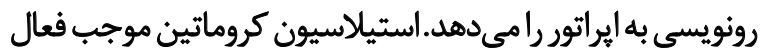

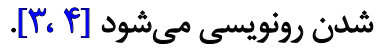
هيستون داستيلازها شامل كروهى از آنزيمها هستند كه مسئول حذف كروههاى استيل از اسيدآمينه ليزيناندا آندينا تغييرات

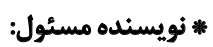

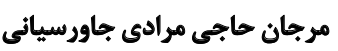

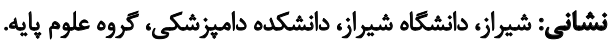

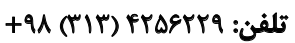
يست الكترونيكي: marhaji@yahoo.com 


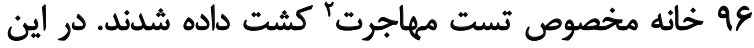

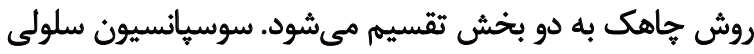

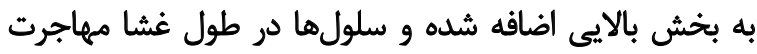

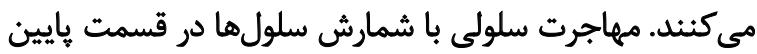

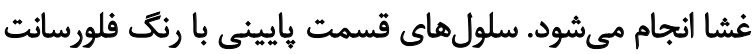

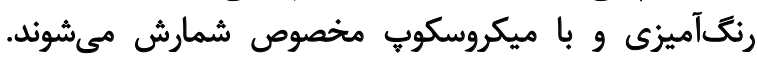

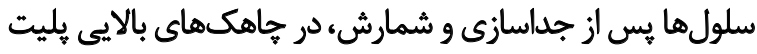

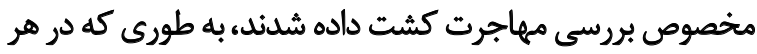

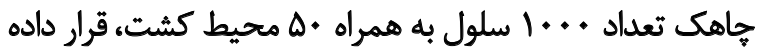

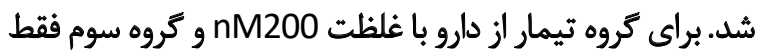

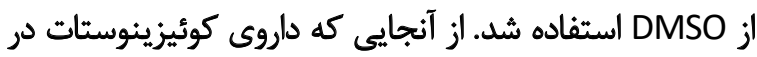
Dل مي شود و با توجه به سميت DMSO

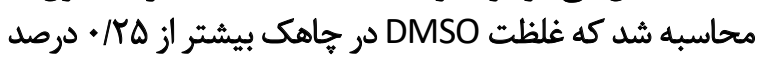

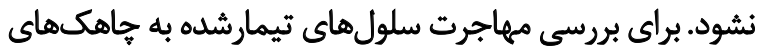

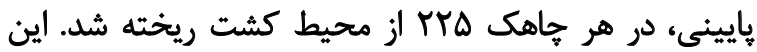

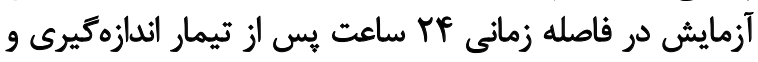

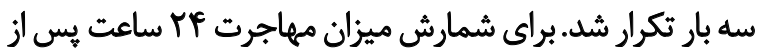

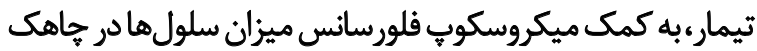

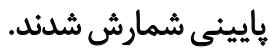

ياقتهها

قبل از اضافه كردن سوسيانسيون سلولى به قسمت بالايى

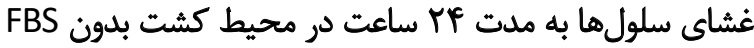

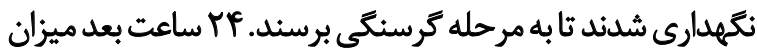

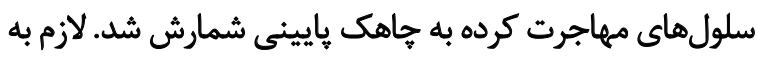

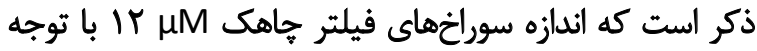

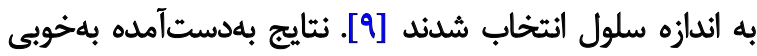

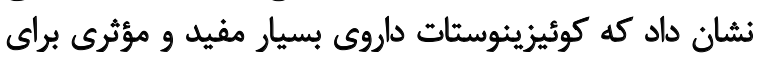

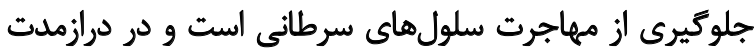

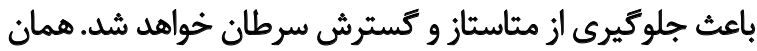

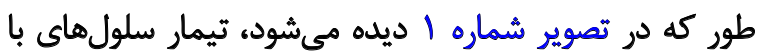

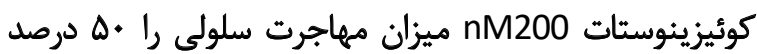
كاهش داده است (تصوير شماره )).

تمام آزمايشها حداقل سه بار تكرار شد. دادهها به كمك آند

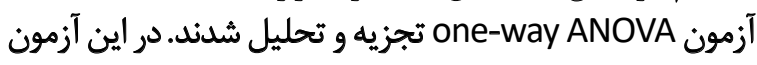
مقدار ه ٪ • يا كمتر معنى دار در نظر كرفته شد.

بحث

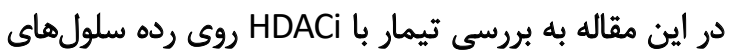

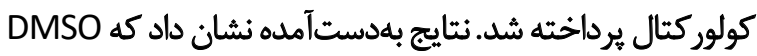

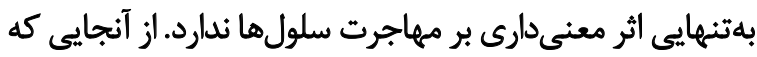

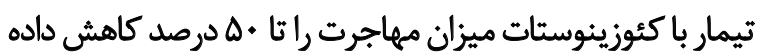

انتخابي كه در اركان هدف مدنظر است را به طور مطلوب نداشته

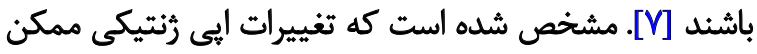

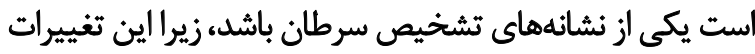

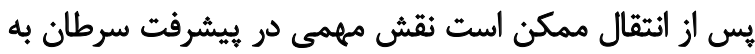

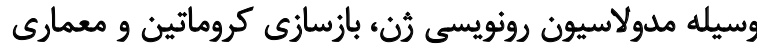

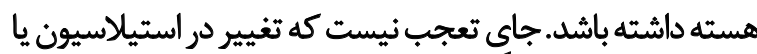

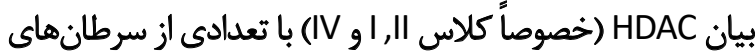

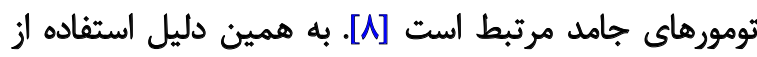

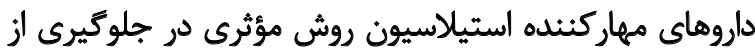

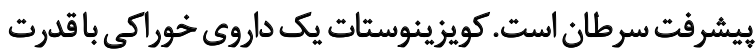

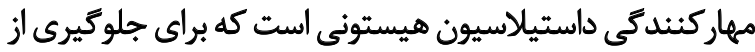

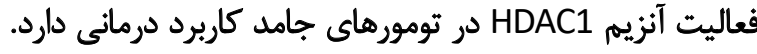

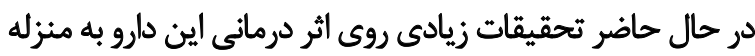

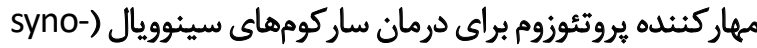

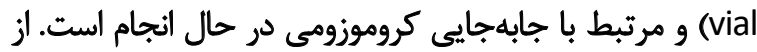

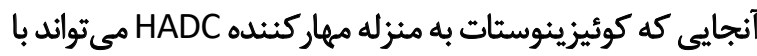

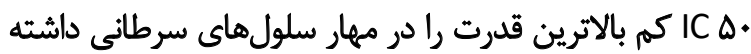

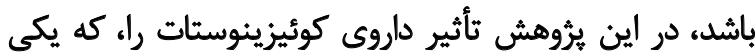

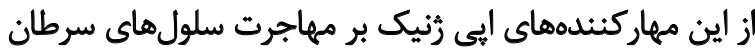
كولوركتال است، بررسى مى كنينه.

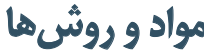

سلولهاى اوليه جداشده از بافت سرطانى كولون از بيوبانك

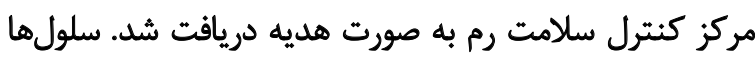

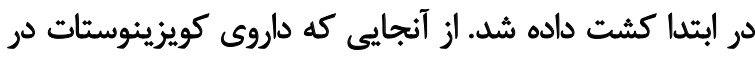

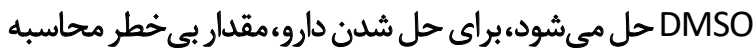

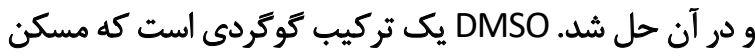

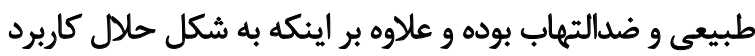

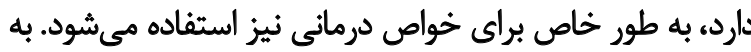

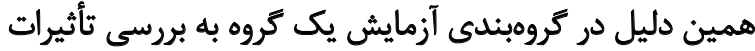

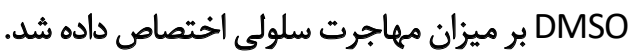

براى بررسى تركيبات موجود در نيج كه مي تواند بر مهاجرت

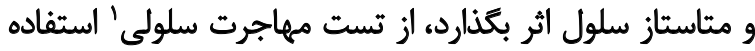

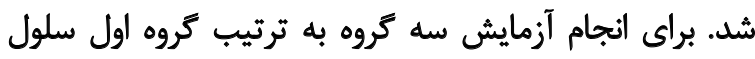

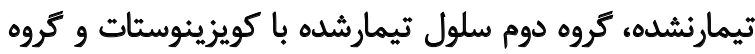

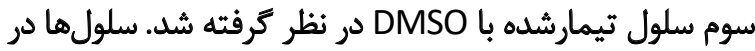

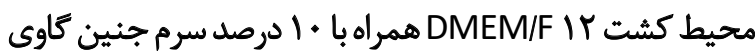

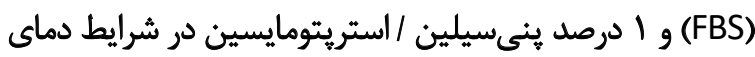

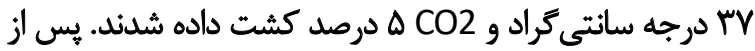

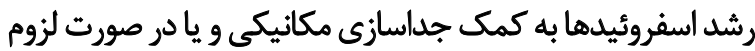

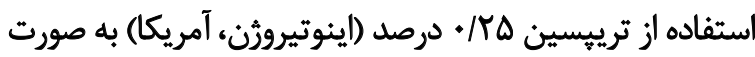

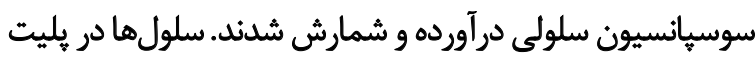




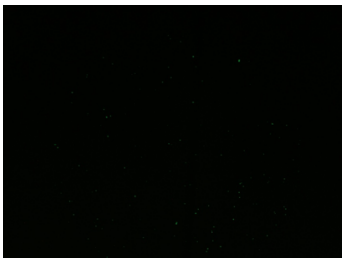

Control

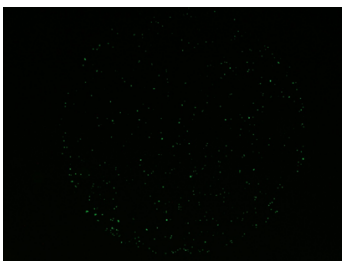

Q treated

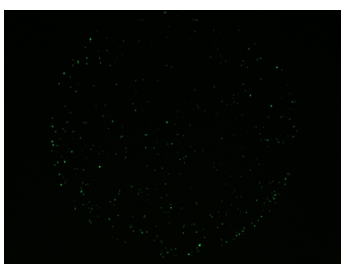

DMSO

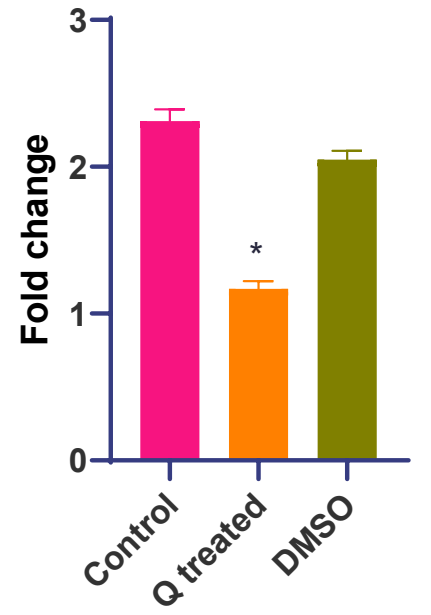

Control

$Q$ treated

DMSO

تصوير ا. سلولهاى سرطانى كاشته شده در يليت مخصوص آزمايش مهاجرت سلولى

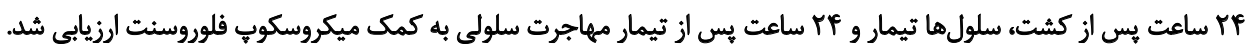

ايجاد اهداف درمانى و بالاخص دارويى جديد در درمان سرطانها

فراهم شده است [ن]

بررسى و ترميم استيلاسيون هيستونها به وسيله مولكول هاييى كه مانع از فعاليت نابهجاي هيستون نداستيلاز ها مي شود، مي تواند نقش مهمى در هومئوستازى و عملكرد يروتئين هائ كروماتين

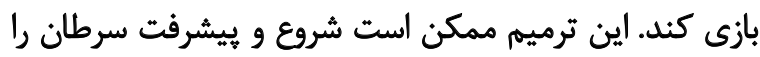

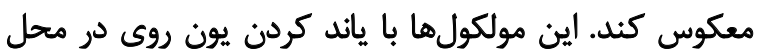

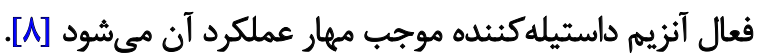

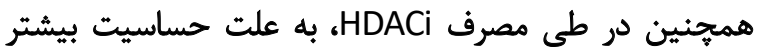

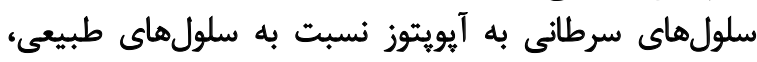

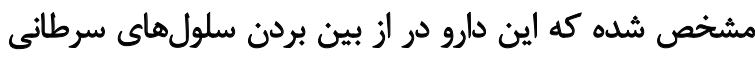

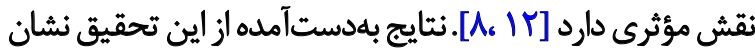

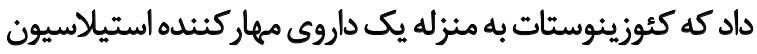

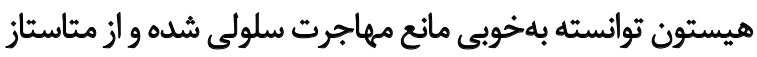
سرطان جلوكيرى كند.

\section{$5,5 d x$}

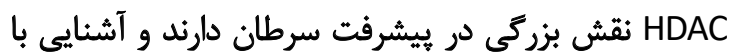

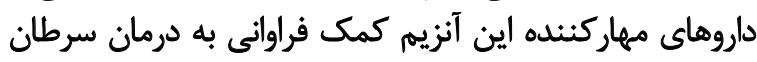

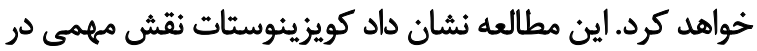
مهار اين آتزيم دارد.
است، مى توان نتيجه كرفت كئوزينوستات به وسيله داستيلاسيون

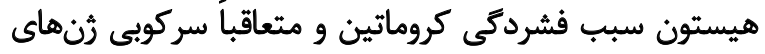

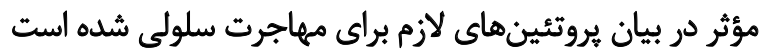

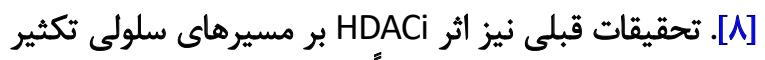

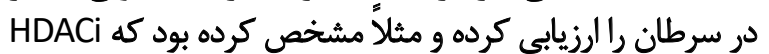

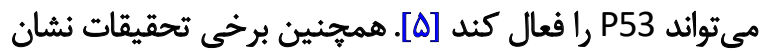
داده است كه در حضور

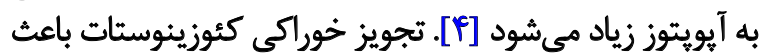

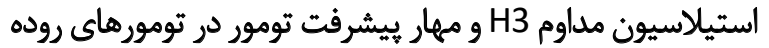

بزرگ ميىشود [ب]. انزيم هيستون داستيلاز ميتواند بر ميزان تراكم كروماتين تأثير بحنارد و از طريق دي استيل كردن باقي ماندهائي ليزين،

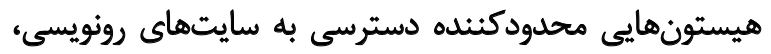

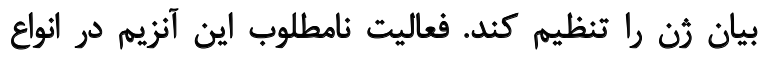

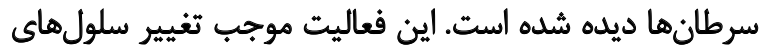

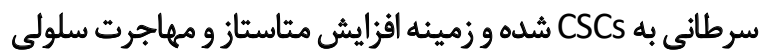

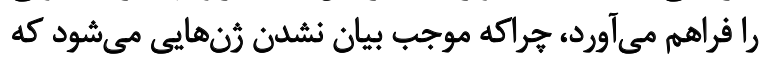

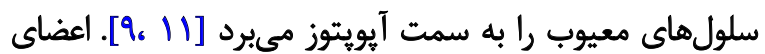

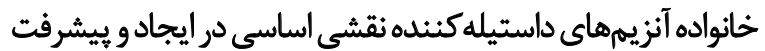

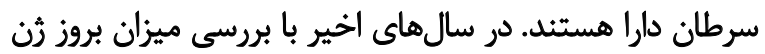

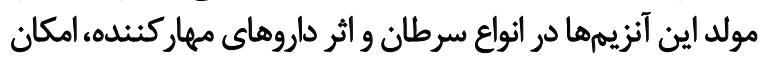




\section{ملاحظات اخلاقي}

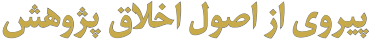

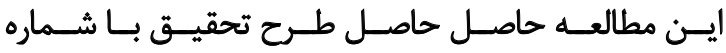

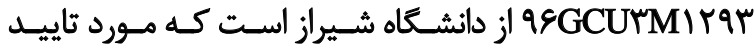

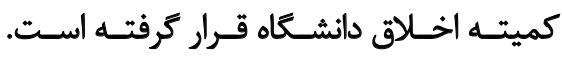

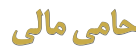

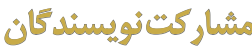

$$
\begin{aligned}
& \text { تمام نويسندكان در طراحي، اجرا و نكارش همه بخشهاي } \\
& \text { ئروهش حاضر مشاركت داشتهاند. }
\end{aligned}
$$

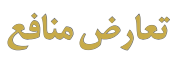

بنابر اظهار نويسندكان اين مقاله تعارض منافع ندارد.

$$
\text { 管 }
$$

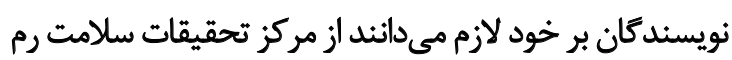

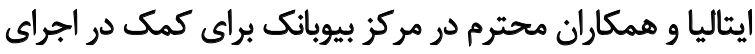
اين يروره تشكر و قدردانى كنيند. 


\section{References}

[1] Torre LA, Bray F, Siegel RL, Ferlay J, Lortet-Tieulent J, Jemal A. CA: Global cancer statistics. Am Cancer Soc J. 2012; 65(2):87-108. [DOI:10.3322/ caac.21262]

[2] Fiori ME, Villanova L, De Maria R. Cancer stem cells: At the forefront of personalized medicine and immunotherapy. Curr Opin Pharmacol. 2017; 35:1-11. [DOI:10.1016/j.coph.2017.04.006]

[3] Carol H, Gorlick R, Kolb EA, Morton CL, Moradi Manesh D, Keir ST, et al. Initial testing (stage 1) of the histone deacetylase inhibitor, quisinostat (JNJ-26481585), by the Pediatric Preclinical Testing Program. Pediatr Blood Cancer. 2014; 61(2):245-52. [DOI:10.1002/pbc.24724]

[4] Arts J, King P, Mariën A, Floren W, Beliën A, Janssen L, et al. JNJ26481585, a novel "second-generation" oral histone deacetylase inhibitor, shows broad-spectrum preclinical antitumoral activity. Clin Cancer Res. 2009; 15(22):6841-51. [DOI:10.1158/1078-0432.CCR-09-0547]

[5] Bose P, Dai Y, Grant S. Histone deacetylase inhibitor (HDACl) mechanisms of action: Emerging insights. Pharmacol Ther. 2014; 143(3):323-36. [DOI:10.1016/j.pharmthera.2014.04.004]

[6] Venugopal B, Baird R, Kristeleit RS, Plummer R, Cowan R, Stewart A, et al. A phase I study of quisinostat (JNJ-26481585), an oral hydroxamate histone deacetylase inhibitor with evidence of target modulation and antitumor activity, in patients with advanced solid tumors. Clin Cancer Res. 2013; 19(15):4262-72. [DOI:10.1158/1078-0432.CCR-13-0312]

[7] de Nadal E, Zapater M, Alepuz PM, Sumoy L, Mas G, Posas F. The MAPK Hog1 recruits Rpd3 histone deacetylase to activate osmoresponsive genes. Nature. 2004; 427:370-4. [DOI:10.1038/nature02258]

[8] Li Y, Seto E. HDACs and HDAC inhibitors in cancer development and therapy. Cold Spring Harb Perspect Med. 2016; 6:a026831. [DOI:10.1101/ cshperspect.a026831]

[9] Hulkower KI, Herber RL. Cell migration and invasion assays as tools for drug discovery. Pharm. 2011; 3(1):107-24.[DOI:10.3390/pharmaceutics3010107]

[10] Wainwright EN, Scaffidi P. Epigenetics and cancer stem cells: Unleashing, hijacking, and restricting cellular plasticity. Trends Cancer. 2017; 3(5):372-86. [DOI:10.1016/j.trecan.2017.04.004]

[11] Heijkants R, Willekens K, Schoonderwoerd M, Teunisse A, Nieveen M, Radaelli $E$, et al. Combined inhibition of CDK and HDAC as a promising therapeutic strategy for both cutaneous and uveal metastatic melanoma. Oncotarget. 2018; 9:6174-87. [DOI:10.18632/oncotarget.23485]

[12] Guenther MG, Barak OR, Lazar MA. The SMRT and N-CoR corepressors are activating cofactors for histone deacetylase 3. Mol Cell Biol. 2001; 21(18):6091-101. [DOI:10.1128/MCB.21.18.6091-6101.2001] 